\title{
Vibration Reliability Analysis of Drum Brake Using the Artificial Neural Network and Important Sampling Method
}

\author{
Zhou Yang $\mathbb{D}^{1},{ }^{1}$ Unsong Pak, ${ }^{1,2}$ and Cholu Kwon ${ }^{1,3}$ \\ ${ }^{1}$ School of Mechanical Engineering \& Automation, Northeastern University, Shenyang 110819, China \\ ${ }^{2}$ Pyongyang Transportation University, Pyongyang 999093, Democratic People's Republic of Korea \\ ${ }^{3}$ Institute of Mechanical Engineering State Academy of Sciences, Pyongyang 999093, Democratic People's Republic of Korea \\ Correspondence should be addressed to Zhou Yang; yangzhou@mail.neu.edu.cn
}

Received 22 February 2021; Revised 29 April 2021; Accepted 13 May 2021; Published 25 May 2021

Academic Editor: Adil Mehmood Khan

Copyright ( $\odot 2021$ Zhou Yang et al. This is an open access article distributed under the Creative Commons Attribution License, which permits unrestricted use, distribution, and reproduction in any medium, provided the original work is properly cited.

This research aims to evaluate the calculation accuracy and efficiency of the artificial neural network-based important sampling method (ANN-IS) on reliability of structures such as drum brakes. The finite element analysis (FEA) result is used to establish the ANN sample in ANN-based reliability analysis methods. Because the process of FEA is time-consuming, the ANN sample size has a very important influence on the calculation efficiency. Two types of ANNs used in this study are the radial basis function neural network (RBF) and back propagation neural network (BP). RBF-IS and BP-IS methods are used to conduct reliability analysis on training samples of three different sizes, and the results are compared with several reliability analysis methods based on ANNs. The results show that the probability of failure of the RBF-IS method is closer to that of the Monte-Carlo simulation method (MCS) than those of other methods (including BP-IS). In addition, the RBF-IS method has better calculation efficiency than the other methods considered in this study. This research demonstrates that the RBF-IS method is well suited to structure reliability problems.

\section{Introduction}

Since brakes serve the essential purpose of slowing down and stopping vehicles, the reliability of brakes significantly impacts vehicle safety. Due to their advantages of large braking torque and low cost, drum brakes are widely used in automobiles [1-3]. Resonance occurs when the driving frequency is close to the natural frequency of the brake and can cause fatigue damage to the brakes and other components, potentially leading to failure of the braking system. Therefore, research on vibration reliability analysis of brake component is necessary.

Over the years, many scholars have contributed to significant research on structure reliability analysis. The most common methods used for reliability analysis include the first-order reliability method (FORM), second-order reliability method (SORM), and MCS. FORM [4-6] and SORM [6-8] of the approximation method are widely used for the reliability analysis due to the simplicity and efficiency of these methods. In addition, because the MCS method is very simple and easy to program, it is widely used for the reliability analysis of the engineering structures. However, this method requires a significant computation effort for low probability of failure (POF) problem [9]. In order to address this issue, several methods have been proposed to reduce computation effort, such as the IS [10, 11], directional importance sampling simulation method [12], and subset simulation method $[13,14]$.

Since the limit state function (LSF) of most engineering structures is generally either nonlinear or implicit, the FEA method is required to compute structure responses, such as deformations, frequencies, and stresses. FEA is often combined with reliability analysis methods to conduct the engineering structure reliability analysis. While it is easy to combine FEA with the MCS method to conduct reliability analysis, this method is computationally expensive. In recent years, metamodeling techniques have been developed to overcome this issue, such as the model tree (MT), 
evolutionary polynomial regression (EPR), multivariate adaptive regression spline (MARS), gene expression programming (GEP) [15], response surface method (RSM) [16-18], support vector machine [19, 20], kriging surrogate model [21-24], and ANN [25-32]. Metamodeling techniques are adopted to establish the approximate models, which can replace the original implicit LSF. The ANN is widely used to solve reliability problems related to practical engineering structure. In this setup, FEA is used to obtain training sample of the ANN, the random variables of the structure are used for ANN input, the structure response is used for ANN output, and explicit form of the function relationship between the structure response and random variables can be established. The BP-based MCS method is used to predict the structure reliability, and the result is compared with three reliability analysis methods including the traditional MCS, polynomial-FOSM, and BP-based advanced first-order second moment method (AFOSM) [27]. The combination of an adaptive RBF metamodeling technique and a FORM proposes a new reliability analysis method for the practical tunnel engineering problems [28]. The uniform design method is used to improve the disadvantage of the conventional ANN-based RSM method [29]. The development and use of ANN-based reliability analysis methods is surveyed to propose the improvement techniques of the ANN training set [30]. Two ANNs (including a multilayer perceptron network and RBF) based several reliability analysis methods is used to conduct a reliability analysis of the laminated composite structures [31]. In the ANN-based reliability analysis method, the most of the calculation time is spent conducting the FEA, and the ANN sample data size has a significant influence on the calculation efficiency of the ANN-based reliability analysis method.

This study evaluates the calculation accuracy and efficiency of the ANN-IS method on reliability of structures. Using two numeric verification problems and a drum brake vibration reliability problem as examples, the RBF-IS method and BP-IS method are used to conduct the reliability analysis on the training samples equal to 100,200 , and 300 units. The calculation results are compared with several methods including MCS, AFOSM, BP-MCS, BP-AFOSM, RBF-MCS, and RBF-AFOSM.

This study is structured as follows: Section 2 presents a vibration reliability analysis based on the ANN-IS method, Section 3 provides the numerical analyses and results, and Section 4 presents the discussion and conclusions.

\section{Vibration Reliability Analysis Based on the ANN-IS Method}

2.1. Basis Theory of the Vibration Reliability Analysis. Resonance occurs when the driving frequency is close to the natural frequency of a structure, such that the structure enters a quasifailure state $[33,34]$. According to reliability theory, the state function of the structure with vibration is defined as

$$
G(f, w)=|f-w|
$$

where $f$ is the natural frequency of the structure, and $w$ is the driving frequency.

Based on the relationship between the structure natural frequency $f$ and the driving frequency $w$, the state function of the quasifailure can be expressed as

$$
G(f, w)=|f-w| \leq \varepsilon
$$

where $\varepsilon$ is the specified range, in which the value is generally between 0.1 and 0.15 times the natural frequency of structure.

The mean value $\mu_{G}$ and variance $\sigma_{G}^{2}$ of the state function can be expressed as

$$
\begin{aligned}
\mu_{G} & =E(G) \\
& =|E(f)-E(w)|, \\
\sigma_{G}^{2} & =\operatorname{Var}(G) \\
& =\sigma_{f}^{2}+\sigma_{w}^{2} .
\end{aligned}
$$
as

The quasifailure probability of the structure is expressed

$$
P_{F}=P(-\varepsilon \leq f-w \leq \varepsilon) .
$$

If the natural frequency and the driving frequency are independent normally distributed, then the probability of failure can be expressed as

$$
P_{F}=\Phi\left(\frac{\varepsilon-\mu_{G}}{\sigma_{G}}\right)-\Phi\left(\frac{-\varepsilon-\mu_{G}}{\sigma_{G}}\right) .
$$

2.2. Importance Sampling Method. The importance sampling method is a commonly improved MCS method. This method is widely used because of its high sampling efficiency and small variance. The basic theory of this method is that the importance sampling density function is adopted to replace the original probability density function. The POF base on the IS method is computed as

$$
\begin{aligned}
P_{F} & =\int_{R^{m}} I_{F}(X) f(X) \mathrm{d} X \\
& =\int_{R^{m}} I_{F}(X) \frac{f(X)}{h(X)} h(X) \mathrm{d} X \\
& =E\left[I_{F}(X) \frac{f(X)}{h(X)}\right],
\end{aligned}
$$

where $P_{F}$ is the POF of the reliability performance function, $h(X)$ is the importance sampling density function, $f(X)$ is the joint probability density function of random variables, $R^{m}$ is the m-dimension variable space, and $I_{F}(X)$ is the indicator function of failure domain.

$$
I_{F}(X)= \begin{cases}0, & G(X) \leq 0 \\ 1, & G(X)>0 .\end{cases}
$$

The estimated value $\left(\widehat{P}_{F}\right)$ of POF is computed as 


$$
\widehat{P}_{F}=\frac{1}{N} \sum_{i=1}^{N}\left[I_{F}\left(X_{i}\right) \frac{f\left(X_{i}\right)}{h\left(X_{i}\right)}\right]
$$

The mean value, variance, and coefficient of variation $(\mathrm{COV})$ of the estimated failure probability are computed as

$$
\begin{aligned}
E\left[\widehat{P}_{F}\right] & =E\left\{\frac{1}{N} \sum_{i=1}^{N}\left[I_{F}\left(X_{i}\right) \frac{f\left(X_{i}\right)}{h\left(X_{i}\right)}\right]\right\} \\
& =E\left[I_{F}(X) \frac{f(X)}{h(X)}\right] \\
& =P_{F} . \\
\operatorname{Var}\left[\widehat{P}_{F}\right] & =\frac{1}{N} \operatorname{Var}\left[I_{F}(X) \frac{f(X)}{h(X)}\right] \\
& \approx \frac{1}{N-1}\left\{\frac{1}{N} \sum_{i=1}^{N}\left[I_{F}\left(X_{i}\right) \frac{f^{2}\left(X_{i}\right)}{h^{2}\left(X_{i}\right)}\right]-\widehat{P}_{F}^{2}\right\}, \\
\operatorname{Cov}\left[\widehat{P}_{F}\right] & =\frac{\sqrt{\operatorname{Var}\left[\widehat{P}_{F}\right]}}{E\left[\widehat{P}_{F}\right]} .
\end{aligned}
$$

2.3. Artificial Neural Network. When the reliability analysis method is used to for a complex structure, the responses of the structure need to be calculated by sophisticated numerical methods, such as FEA. However, this method is not suitable for reliability analysis of a complex structure because of its long computation time. In order to reduce the computation time, the ANN is commonly used to establish the LSF.

2.3.1. BP Neural Network. BP neural network is a widely used type of the ANN. The structure of a BP neural network is shown in Figure 1.

In this study, the BP model includes a hidden layer, an input layer, and an output layer, where the design parameters (material properties and structural dimensions) of drum brake are used for input data, and the natural frequency of the drum brake is used for output data. According to references $[27,35,36]$, the number of neurons in the hidden layer is calculated as

$$
p=\sqrt{n=m}+a,
$$

where $p$ is the neuron number of the hidden layer, $n$ is the neuron number of the input layer, $m$ is the neuron number of the output layer, and $a$ is an adjusting constant; according to references $[27,35,36]$, the range of $a$ is the integer between 1 and 10.

The output model of the hidden layer neuron is computed as

$$
\mathrm{Q}_{j}=f\left(\sum \mathrm{IW}_{\mathrm{ij}} \times X_{i}-b_{j}\right),
$$

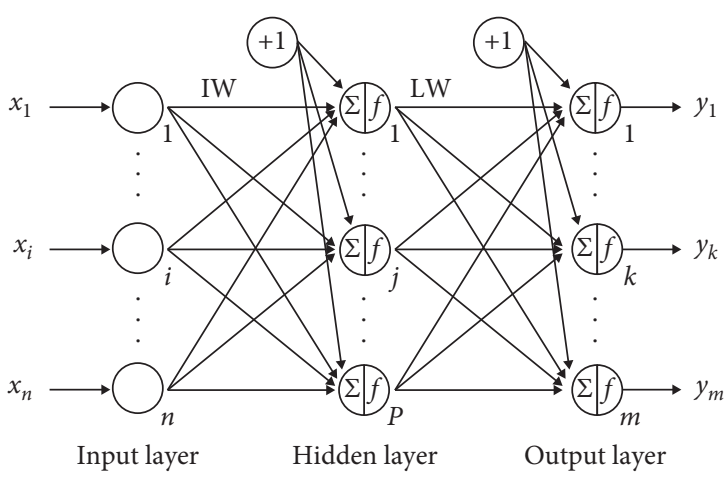

FIgURE 1: BP neural network.

where IW is the weight matrix between the input layer and the hidden layer, $b$ is the threshold matrix, $X$ is the vector of input variables, and $f()$ is the neuron transfer function between the input layer and hidden layer. Logsig and tansig functions are widely used in the transfer function.

The output model of the output layer neuron is computed as

$$
G_{k}=f\left(\sum \mathrm{LW}_{\mathrm{jk}} \times Q_{j}-b_{k}\right),
$$

where LW is the weight matrix between the hidden layer and the output layer, $b$ is the threshold matrix, and $f$ is the neuron transfer function between the hidden layer and output layer. Purelin function is widely using in the transfer function.

If the difference between the model output value and the expected value exceeds the expected error value, gradient search technique is used to adjust the weight and threshold values, and the output value is recalculated. This process is repeated until the difference between the model output value and expected value is found to be less than the expected error value.

2.3.2. RBF Neural Network. RBF is a two-layer forward neural network. The RBF model includes the input layer, output layer, and one hidden layer. The RBF neural network structure model is shown in Figure 2.

RBF typically uses the Gaussian function. The activation function is computed as

$$
G\left(X-a_{j}\right)=\exp \left(-\frac{1}{2 * \sigma^{2}} X-a_{j}^{2}\right),
$$

where $X-a_{j}$ is the Euclidean norm, $a_{j}$ is the Gaussian function center, $\sigma$ is the Gaussian function variance, and $j=$ $1,2, \ldots, p$ is the hidden layer neuron number.

The output function of the RBF model is computed as

$$
y_{k}=\sum_{j=1}^{p} w_{\mathrm{jk}} \exp \left(-\frac{1}{2 * \sigma^{2}} X-a_{j}^{2}\right),
$$

where $X=\left(x_{1}, \ldots, x_{i}, \ldots, x_{n}\right)^{T}$ is the input parameters vector, $k=1,2, \ldots, m$ is the output layer neuron number, and $w_{\mathrm{jk}}$ is the weight matrix between the hidden layer and the output layer.

The variance of basis function is computed as 


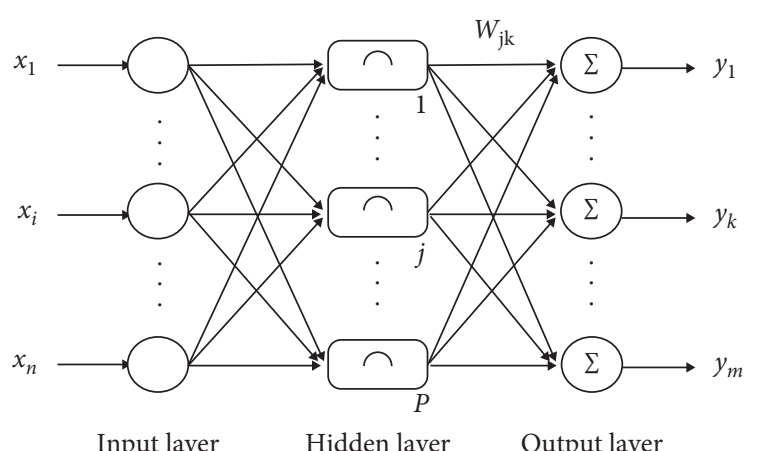

Figure 2: RBF neural network.

$$
\sigma=\frac{1}{s} \sum_{k=1}^{m} d_{k}-y_{k} * a_{j}^{2},
$$

where $d$ is the expected output value, and $s$ is the sample number. RBF has a faster training speed.

2.4. Vibration Reliability Analysis Method of the Drum Brake Based on ANN-IS. The ANN-IS method is used to conduct the drum brake vibration reliability analysis based on the theory in Section 2.1 using ANN samples of different sizes. The steps of the method are given (for a detailed flowchart of the ANN-IS method, Figure 3.) Pseudocode of the proposed method can be viewed from the Supplementary Data 1.

(1 )Define the ANN sample data size $N_{0}$. In this study, $N_{0}=100,200,300$.

(2) The design of the experiment method is used to generate ANN sample points, and FEA is adopted to obtain the natural frequency of the drum brake at the training sample points.

(3) According to the relation criterion in which the difference between the natural frequency and driving frequency of the drum brake could not exceed the specific value, the ANN is used to establish the LSF. In this study, the specific value is defined 0.1 times of the natural frequency.

(4 )The optimization algorithm such as AFOSM is used to evaluate the most probable point $X^{*}$ of the LSF

(5) The important sampling density function $h(X)$ is constructed with the most probable point as the sampling center

(6 ) Define the sample size $N_{1}=1 E+4$. Start iteration $k=1$, according to the important sampling density function, generate sample point of the input variables $X_{k}=\left(x_{1}, x_{2}, \ldots, x_{n}\right)$.

(7 )Trained ANN is used to calculate the LSF value at the sample point $X_{k}$, and indicator function value $I_{F}\left(X_{k}\right)$ calculates using equation (7). The Num1 and Num2 are calculated.

(8) If the iteration number $k=N_{1}$, go to step 9. If not, iteration number $k=k+1$, go to step 6 .
(9) The estimated value of the POF $\left(\widehat{P}_{F}\right)$ and COV of the POF is calculated using equation (8) and equation (9).

\section{Numerical Analyses and Results}

To evaluate the calculation accuracy and efficiency of the proposed reliability analysis method, three examples are used to conduct the reliability analysis including the drum brake problem and two verification problems from the reference literature. In this study, BP and RBF neural networks are used to establish the LSF, and the same sample data are used for two neural networks. ANN sample data size is defined as $N_{0}=100,200,300$, and the ANN sample data are divided into training data and testing data of the ANN [35-37] where $90 \%$ of ANN sample data are used for training data, and $10 \%$ of ANN sample data is used for testing data. In order to evaluate the error of ANNs more accurately, the same testing sample data are used to compare the mean square errors (MSEs) of three ANN sample data $\left(N_{0}=100,200,300\right)$, in which the testing sample data have 10 sample points.

3.1. Case 1: First Verification Problem. The LSF with two random variables is as follows [17]:

$$
G(X)=\exp \left(0.2 x_{1}+1.4\right)-x_{2},
$$

where $X=\left(x_{1}, x_{2}\right)$ is the random variables of standard normal distribution.

ANN sample data sizes are defined such as $N_{0}=100,200,300$. ANN sample points are generated to calculate the original LSF values. The trained ANNs are used to establish the LSF under three training samples of difference sizes, respectively. The same training sample is used for both RBF and BP. Figure 4 shows the comparison between the original LSF values and the predicted LSF values by ANNs. The maximum error point of the testing sample is the third sample point.

The ANN sample sizes are 100, 200, and 300, the mean square errors (MSEs) of RBF are $4.03 E-7,9.81 E-10$, and $1.82 E-10$; the MSEs of BP are $6.99 E-6,1.37 E-6$, and $4.44 E-7$, respectively. Figure 5 shows the comparison of the MSE between RBF and BP.

The result shows the MSE of RBF is smaller than that of $\mathrm{BP}$. In addition, the MSE of RBF is already very small for an ANN sample size of 200. Define the number of samples, in this study, $N_{1}=1 E+4$. Two ANN-IS methods and several reliability analysis methods including AFOSM, IS, BP-MCS, BP-AFOSM, RBF-MCS, and RBF-AFOSM are used to calculate the reliability indices on training sample of three difference sizes. The calculated results are compared with the MCS method. The result is given in Tables 1-3 and Figure 6.

The POF of the MCS, AFOSM, and IS is $3.58 E-04$, $4.05 E-04$, and $3.58 E-04$, respectively. The POF of the RBF-IS method is $3.59 E-04,3.59 E-04$, and $3.58 E-04$, and the relative errors are $0.3739 \%, 0.1528 \%$, and $0.0058 \%$, respectively. The POF of the BP-IS method is $3.61 E-04$, $3.52 E-04$, and $3.56 E-04$, and relative errors are $0.8292 \%$, 


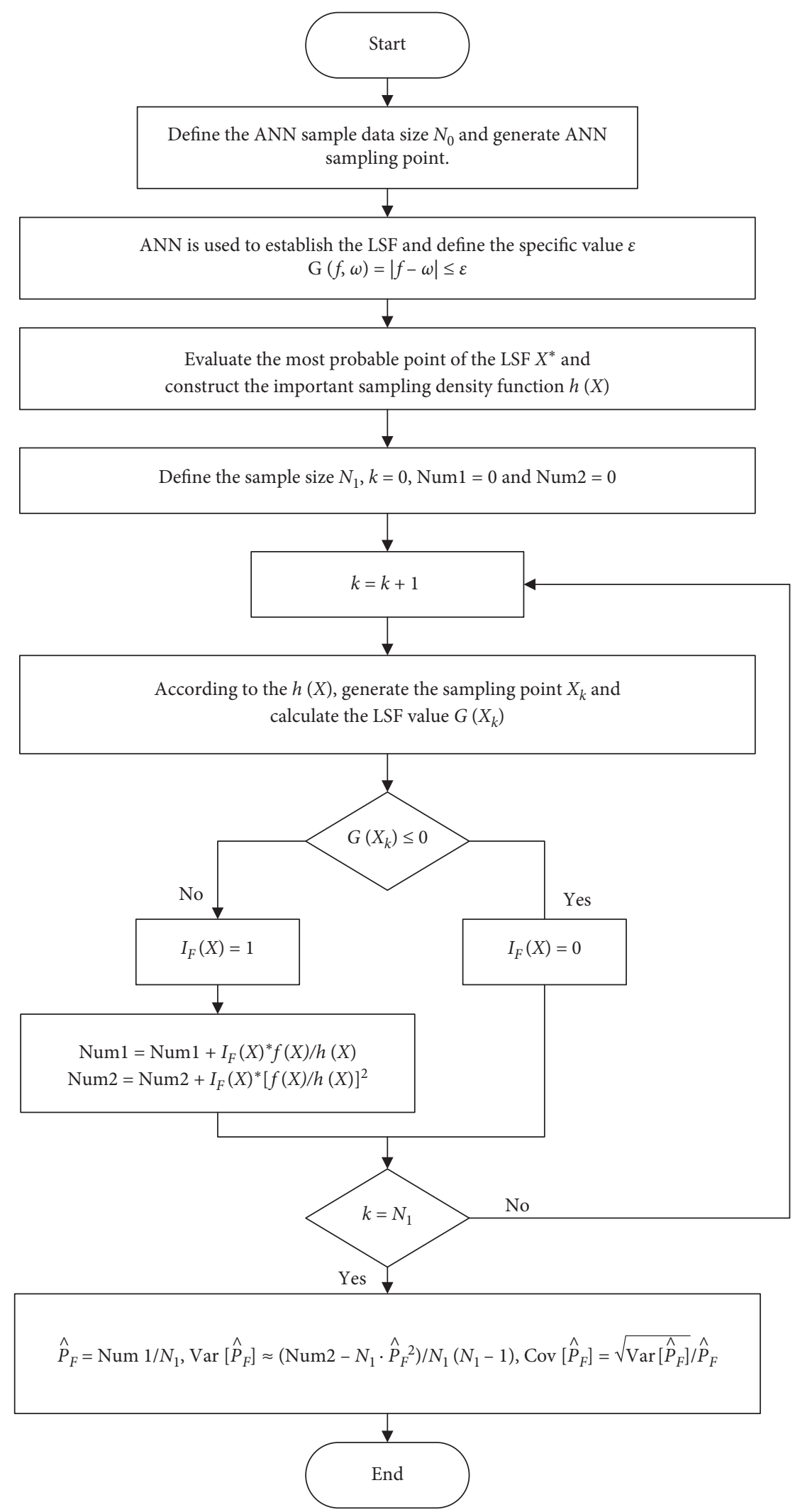

FIgURE 3: The flowchart of reliability analysis of the drum brake based on the ANN-IS method. 


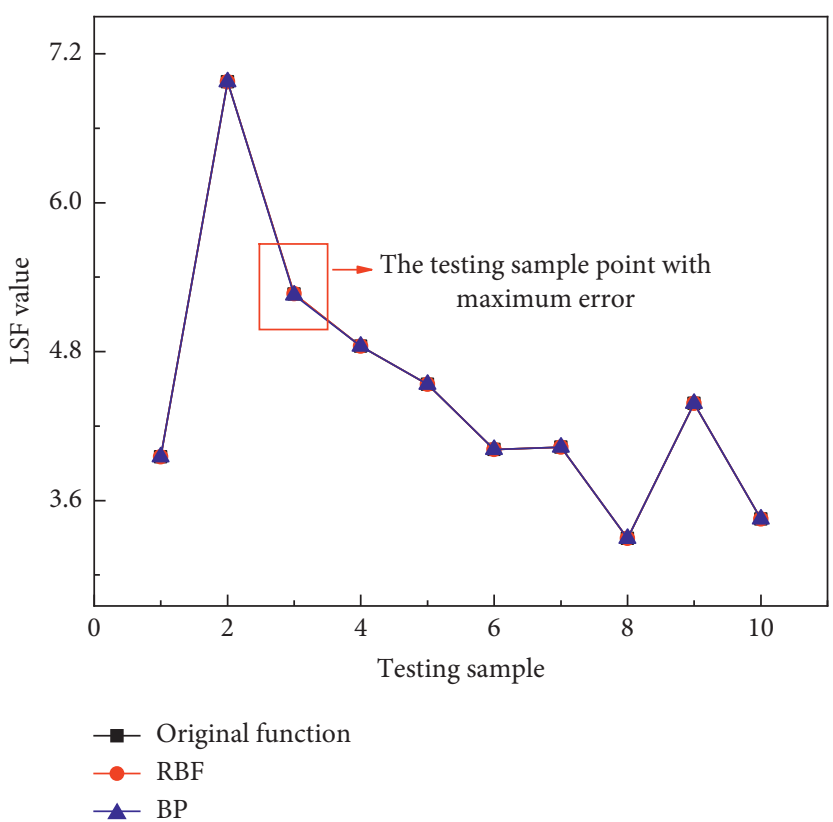

(a)

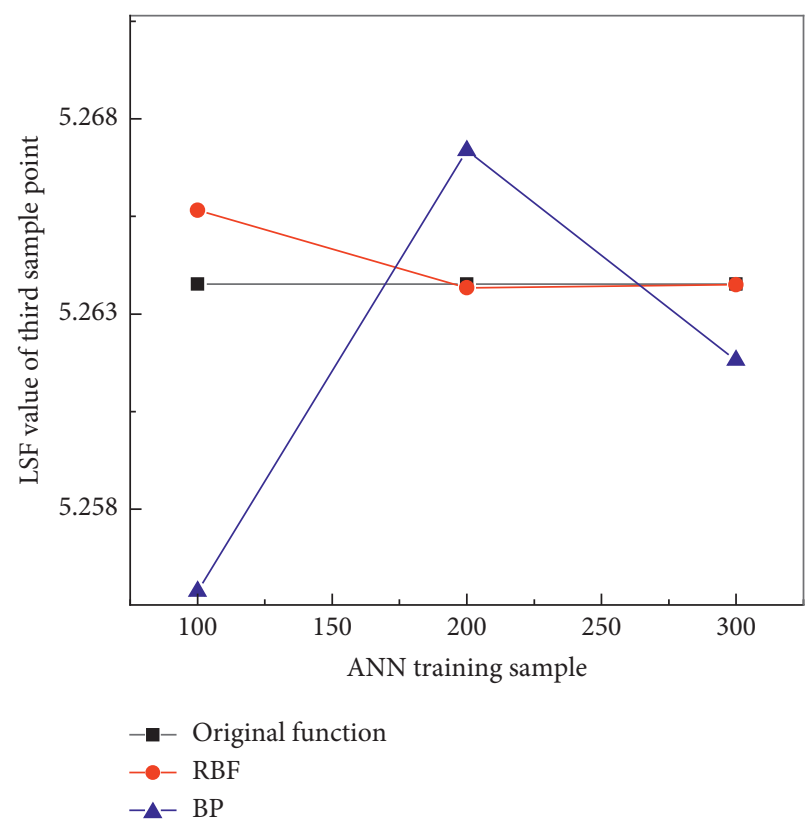

(b)

FIGURE 4: Comparison between the original LSF values and the predicted LSF values by ANNs. (a) The LSF value at testing sample for an ANN sample size of 100. (b) The LSF value of the third testing sample point relative to change in the ANN sample data size.

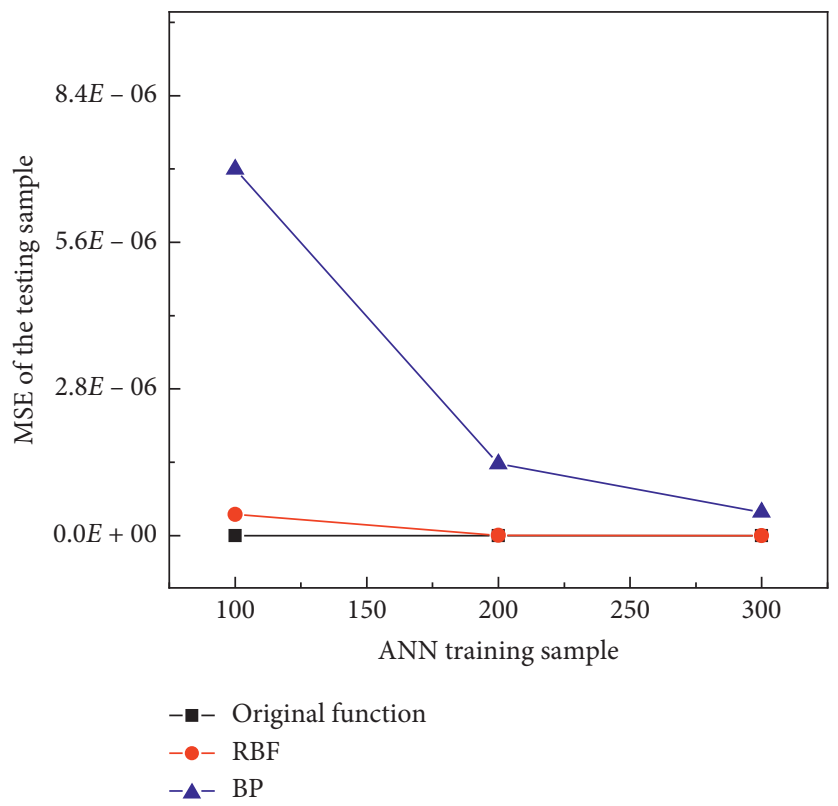

FIgURE 5: Comparison the change of MSE between RBF and BP as the ANN sample data size change.

$1.5901 \%$, and $0.4791 \%$, respectively. The results show that the POF of the RBF-IS method is closer to that of MCS than that of the BP-IS method. In addition, the used of number of samples for RBF-IS is smaller than the ANN-based MCS method, so that the RBF-IS method has better calculation efficiency than the ANN-based MCS method.
3.2. Case 2: Second Verification Problem. The LSF with random variables is as follows [8]; the mean and standard deviation of the random variables is given as Table 4 .

$$
G(X)=\left(1-x_{5} \frac{x_{4} x_{3}}{x_{6} x_{2} x_{7}}\right) x_{4} x_{2} x_{3}-x_{1},
$$


TABLe 1: Comparison of the reliability index and POF from different reliability analysis methods with an ANN sample size of 100.

\begin{tabular}{lcccr}
\hline Method & Reliability index & POF & Number of samples $\left(N_{1}\right)$ & Relative error $(\%)$ \\
\hline MCS & 3.3834 & $3.58 E-04$ & $1 E+7$ & - \\
AFOSM & 3.3497 & $4.05 E-04$ & - & 12.989 \\
IS & 3.3833 & $3.58 E-04$ & $1 E+4$ & 0.0146 \\
BP-MCS & 3.3751 & $3.69 E-04$ & $1 E+7$ & 3.0726 \\
BP-AFOSM & 3.3523 & $4.01 E-04$ & - & 11.914 \\
BP-IS & 3.3811 & $3.61 E-04$ & $1 E+4$ & 0.8292 \\
RBF-MCS & 3.3919 & $3.47 E-04$ & $1 E+7$ & 3.0726 \\
RBF-AFOSM & 3.3531 & $3.99 E-04$ & - & 11.617 \\
RBF-IS & 3.3824 & $3.59 E-04$ & $1 E+4$ & 0.3739 \\
\hline
\end{tabular}

TABLe 2: Comparison of the reliability index and POF from different reliability analysis methods with an ANN sample size of 200.

\begin{tabular}{lcccr}
\hline Method & Reliability index & POF & Number of samples $\left(N_{1}\right)$ & Relative error $(\%)$ \\
\hline MCS & 3.3834 & $3.58 E-04$ & $1 E+7$ & - \\
AFOSM & 3.3497 & $4.05 E-04$ & - & 12.989 \\
IS & 3.3833 & $3.58 E-04$ & $1 E+4$ & 0.0146 \\
BP-MCS & 3.3803 & $3.62 E-04$ & $1 E+7$ & 1.1173 \\
BP-AFOSM & 3.3482 & $4.07 E-04$ & - & 13.607 \\
BP-IS & 3.3878 & $3.52 E-04$ & $1 E+4$ & 1.5901 \\
RBF-MCS & 3.3872 & $3.53 E-04$ & $1 E+7$ & 1.3966 \\
RBF-AFOSM & 3.3501 & $4.04 E-04$ & - & 12.804 \\
RBF-IS & 3.3829 & $3.59 E-04$ & $1 E+4$ & 0.1528 \\
\hline
\end{tabular}

TABle 3: Comparison of the reliability index and POF from different reliability analysis methods with an ANN sample size of 300.

\begin{tabular}{lcccr}
\hline Method & Reliability index & POF & Number of samples $\left(N_{1}\right)$ & Relative error $(\%)$ \\
\hline MCS & 3.3834 & $3.58 E-04$ & $1 E+7$ & - \\
AFOSM & 3.3497 & $4.05 E-04$ & - & 12.989 \\
IS & 3.3833 & $3.58 E-04$ & $1 E+4$ & 0.0146 \\
BP-MCS & 3.3826 & $3.59 E-04$ & $1 E+7$ & 0.2793 \\
BP-AFOSM & 3.3491 & $4.05 E-04$ & - & 13.251 \\
BP-IS & 3.3847 & $3.56 E-04$ & $1 E+4$ & 0.4791 \\
RBF-MCS & 3.3841 & $3.57 E-04$ & $1 E+7$ & 0.2793 \\
RBF-AFOSM & 3.3497 & $4.05 E-04$ & - & 13.001 \\
RBF-IS & 3.3834 & $3.58 E-04$ & $1 E+4$ & 0.0058 \\
\hline
\end{tabular}

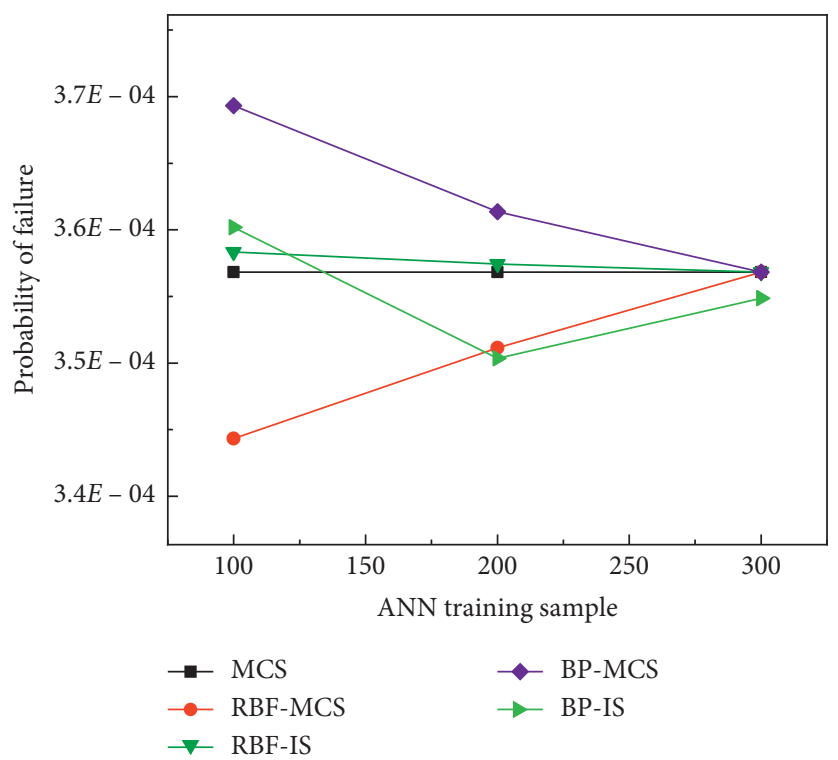

FIgURE 6: Comparison of the POF between ANN-IS (RBF-IS and BP-IS) and various other methods relative to ANN sample data size. 
TABLE 4: The mean and standard deviation of the random variables.

\begin{tabular}{lccc}
\hline Variables & Mean & Standard deviation & Distribution \\
\hline$x_{1}$ & 0.01 & 0.003 & Normal \\
$x_{2}$ & 0.3 & 0.015 & Normal \\
$x_{3}$ & 360 & 36 & Normal \\
$x_{4}$ & $2.26 E-4$ & $1.13 E-5$ & Normal \\
$x_{5}$ & 0.5 & 0.05 & Normal \\
$x_{6}$ & 0.12 & 0.006 & Normal \\
$x_{7}$ & 40 & 6 & Normal \\
\hline
\end{tabular}

where $X=\left(x_{1}, x_{2}, x_{3}, x_{4}, x_{5}, x_{6}, x_{7}\right)$ is the random variable of the normal distribution.

For Case 2, we use the same ANN sample data sizes used in Case 1. The trained RBF and BP are used to establish the LSF on the training samples of three difference sizes, respectively. Figure 7 shows the comparison between the original LSF values and the predicted LSF values by ANNs.

The MSEs of RBF are $1.72 E-10,2.55 E-11$, and $1.49 E-11$; the MSEs of BP are $5.88 E-10,1.59 E-10$, and $2.52 E-11$, respectively. Figure 8 shows the comparison of the MSE between RBF and BP.

The MSEs of RBF are the smaller than that of BP. Two ANN-IS methods and several reliability analysis methods including AFOSM, IS, BP-MCS, BP-AFOSM, RBF-MCS, and RBF-AFOSM are used to calculate the reliability indices on training samples of three difference sizes. The calculated results are compared with the MCS method. The result is given in Tables 5-7 and Figure 9.

The POF of the MCS, AFOSM, and IS is $3.383 E-04$, $3.211 E-04$, and $3.368 E-04$, respectively. The number of samples for MCS method is $1.0 E+7$ and that of ANN-based MCS is $1.0 E+6$. The POF of the RBF-IS method is $3.378 E-04,3.382 E-04$, and $3.383 E-04$, and relative errors are $0.1546 \%, 0.0279 \%$, and $0.0136 \%$, respectively. The POF of the BP-IS method is $3.356 E-04,3.391 E-04$, and $3.381 E-04$, and relative errors are $0.8020 \%, 0.3194 \%$, and $0.0645 \%$, respectively. The results of two verification problems show that the POF of the RBF-IS method is closer to that of the MCS method than that of the BP-IS method. In addition, the number of samples used for the RBF-IS method is smaller than that used for the ANN-based MCS method, demonstrating that the RBF-IS method offers better calculation efficiency than does the ANN-based MCS method. Through two verification problems, the calculation accuracy and efficiency of the proposed method are verified. Therefore, the proposed method is applied to the reliability analysis of the drum brake.

3.3. Application Problem: Vibration Reliability Analysis of the Drum Brake. Resonance will occur when the driving frequency is close to the natural frequency of the brake, and the resonance will cause fatigue damage to the brakes and other components and that may lead to failure of the braking system. In this study, the vibration reliability problem of the drum brake including random parameters is researched. The material properties and structural dimensions of drum brakes are random due to the influence of technical conditions, manufacturing and installation errors, manufacturing environment, and material characteristics. Assuming that the design parameters all obey the normal distribution, the coefficients of variation of the material properties and structural dimensions are determined to be 0.05 and 0.005 . In this study, the density $\left(D_{\mathrm{d}}\right)$, the elastic modulus $\left(E_{\mathrm{d}}\right)$, and Poisson's ratio $\left(P_{\mathrm{d}}\right)$ of the brake drum; the density $\left(D_{\mathrm{s}}\right)$, the elastic modulus $\left(E_{\mathrm{s}}\right)$, and Poisson's ratio $\left(P_{\mathrm{s}}\right)$ of the friction plate; the density $\left(D_{\mathrm{p}}\right)$, the elastic modulus $\left(E_{\mathrm{p}}\right)$, and Poisson's ratio $\left(P_{\mathrm{p}}\right)$ of the brake shoe; width of the brake drum $\left(W_{\mathrm{d}}\right)$, the brake shoe $\left(W_{\mathrm{s}}\right)$, and the friction plate $\left(W_{\mathrm{p}}\right)$; and the initial angle $\left(\theta_{1}\right)$ and envelop angle $\left(\theta_{2}\right)$ of the friction plate are taken as design parameters.

In order to reduce the calculation workload, sensitivity analysis of the design parameters is conducted [15, 35]. Then the "Six sigma analysis" module in the Workbench software is used to conduct the sensitivity analysis on the design parameters of the drum brake. Design of the experiment method is used to generate 50 sample points, and then, modal analysis is conducted to obtain the influence degree of the design parameters on the natural frequency of drum brakes. Figure 10 shows the result of the sensitivity analysis.

Based on the sensitivity analysis results, in order to reduce the calculation workload, the parameters that have little influence on the natural frequency of the brake were screened. Finally, nine parameters were selected as design parameters. The mean and standard deviation of the design parameters is given in Table 8 .

According to the theory of Section 2.1, the LSF of the vibration reliability on the drum brake is as

$$
\mathrm{G}(X)=|f(X)-\omega|<\varepsilon,
$$

where $X=\left(x_{1}, x_{2}, x_{3}, x_{4}, x_{5}, x_{6}, x_{7}, x_{8}, x_{9}\right)$ is the design parameters of the drum brake, and the design parameters are random; $f(X)$ is the natural frequency of the drum brake; $\omega$ is the driving frequency of the drum brake and is the $51 \mathrm{~Hz}$ in this study; and the specified range $\varepsilon$ is 0.1 times of the natural frequency.

Figure 11 shows the FEA result at the mean value of the random parameters.

The ANN sample data sizes are defined such as $N_{0}=100,200,300$, and the design of the experiment method is used to generate training sample points, respectively. FEA is adopted to obtain the natural frequency of drum brake at the training sample points. The ANN sample data can be viewed from the Supplemental Data 2. Trained $\mathrm{RBF}$ and $\mathrm{BP}$ are used to establish the LSF, respectively. Figure 12 shows the comparison between the FEA values and the predicted LSF values by ANNs. 


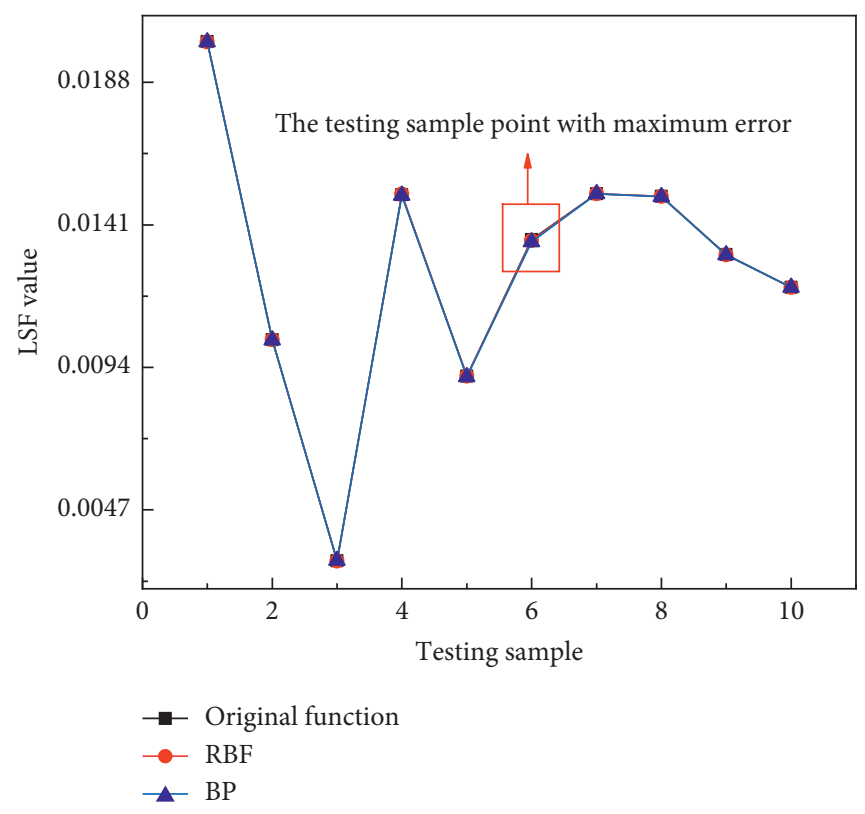

(a)

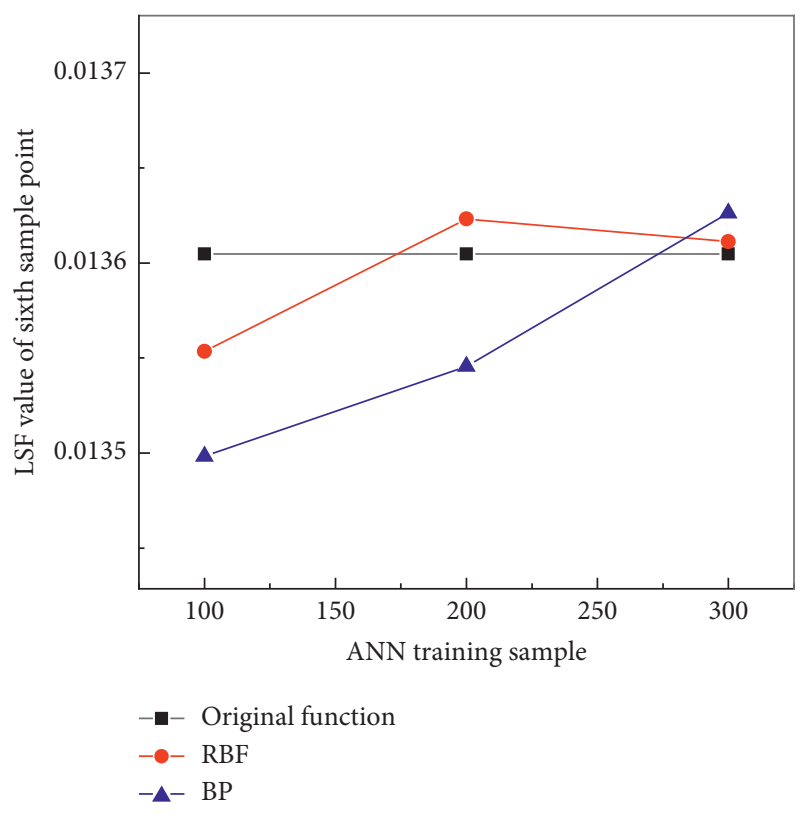

(b)

FIGURE 7: Comparison between the original LSF values and the predicted LSF values by ANNs. (a) The LSF value at testing sample for an ANN sample size of 100. (b) The LSF value of the sixth testing sample point relative to change in the ANN sample data size.

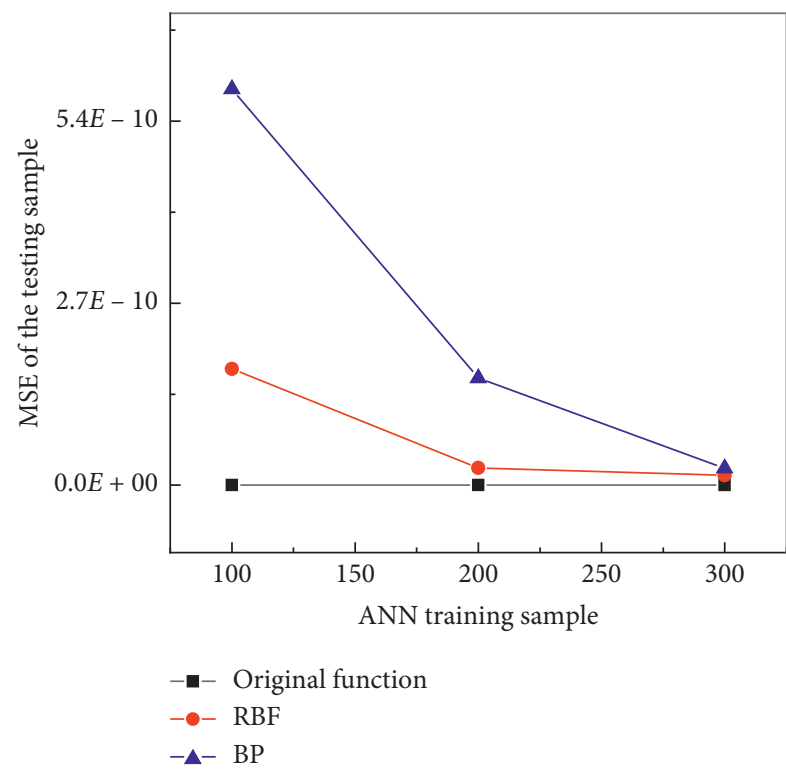

Figure 8: Comparison the change of MSE between RBF and BP as the ANN sample data size change.

The MSEs of RBF are $2.76 E-5,3.95 E-6$, and $1.79 E-6$; the MSEs of BP are $1.05 E-4,2.28 E-5$, and $4.19 E-6$, respectively. Figure 13 shows the comparison the MSE between RBF and BP.

Two ANN-IS methods are used to calculate the reliability indices on training samples of three difference sizes and are compared with ANN-based several reliability analysis methods such as BP-MCS, BP-AFOSM, RBF-MCS, and RBF-AFOSM. The calculated results are given in Tables 9-11 and Figure 14.
TABle 5: Comparison of the reliability index and POF from different reliability analysis methods with an ANN sample size of 100 .

\begin{tabular}{lcccc}
\hline Method & $\begin{array}{c}\text { Reliability } \\
\text { index }\end{array}$ & POF & $\begin{array}{c}\text { Number of } \\
\text { samples }\left(N_{1}\right)\end{array}$ & $\begin{array}{c}\text { Relative } \\
\text { error }(\%)\end{array}$ \\
\hline MCS & 3.3989 & $3.383 E-04$ & $1 E+7$ & - \\
AFOSM & 3.4131 & $3.211 E-04$ & - & 5.0750 \\
IS & 3.4001 & $3.368 E-04$ & $1 E+4$ & 0.4570 \\
BP-MCS & 3.3970 & $3.406 E-04$ & $1 E+7$ & 0.6799 \\
BP- & 3.4117 & $3.228 E-04$ & - & 4.5786 \\
AFOSM & 3.4011 & $3.356 E-04$ & $1 E+4$ & 0.8020 \\
BP-IS & 3.3978 & $3.390 E-04$ & $1 E+7$ & 0.2069 \\
RBF- & & & & \\
MCS & 3.4163 & $3.174 E-04$ & - & 6.1779 \\
RBF- & & & $1 E+4$ & 0.1546 \\
AFOSM & 3.3993 & $3.378 E-04$ & 150 \\
RBF-IS & & & &
\end{tabular}

TABle 6: Comparison of the reliability index and POF from different reliability analysis methods with an ANN sample size of 200.

\begin{tabular}{lcccc}
\hline Method & $\begin{array}{c}\text { Reliability } \\
\text { index }\end{array}$ & POF & $\begin{array}{c}\text { Number of } \\
\text { samples }\left(N_{1}\right)\end{array}$ & $\begin{array}{c}\text { Relative } \\
\text { error }(\%)\end{array}$ \\
\hline MCS & 3.3989 & $3.383 E-04$ & $1 E+7$ & - \\
AFOSM & 3.4131 & $3.211 E-04$ & - & 5.0750 \\
IS & 3.4001 & $3.368 E-04$ & $1 E+4$ & 0.4570 \\
BP-MCS & 3.3987 & $3.385 E-04$ & $1 E+7$ & 0.0591 \\
BP- & 3.4125 & $3.218 E-04$ & - & 4.8719 \\
AFOSM & 3.3983 & $3.391 E-04$ & $1 E+4$ & 0.2310 \\
BP-IS & 3.3991 & $3.380 E-04$ & $1 E+7$ & 0.0887 \\
RBF- & & & & 4.9172 \\
MCS & 3.4127 & $3.217 E-04$ & - & 0.0279 \\
RBF- & 3.3989 & $3.382 E-04$ & $1 E+4$ & \\
AFOSM & & & & \\
RBF-IS & 3.3279 &
\end{tabular}


TABLE 7: Comparison of the reliability index and POF from different reliability analysis methods with an ANN sample size of 300.

\begin{tabular}{lcccc}
\hline Method & Reliability index & POF & Number of samples $\left(N_{1}\right)$ & Relative error $(\%)$ \\
\hline MCS & 3.3989 & $3.383 E-04$ & $1 E+7$ & - \\
AFOSM & 3.4131 & $3.211 E-04$ & - & 5.0750 \\
IS & 3.4001 & $3.368 E-04$ & $1 E+4$ & 0.4570 \\
BP-MCS & 3.3991 & $3.381 E-04$ & $1 E+7$ & 0.0591 \\
BP-AFOSM & 3.4129 & $3.213 E-04$ & - & 5.0216 \\
BP-IS & 3.3991 & $3.381 E-04$ & $1 E+4$ & 0.0645 \\
RBF-MCS & 3.3989 & $3.382 E-04$ & $1 E+7$ & 0.0296 \\
RBF-AFOSM & 3.4129 & $3.214 E-04$ & - & 4.9997 \\
RBF-IS & 3.3989 & $3.383 E-04$ & $1 E+4$ & 0.0136 \\
\hline
\end{tabular}

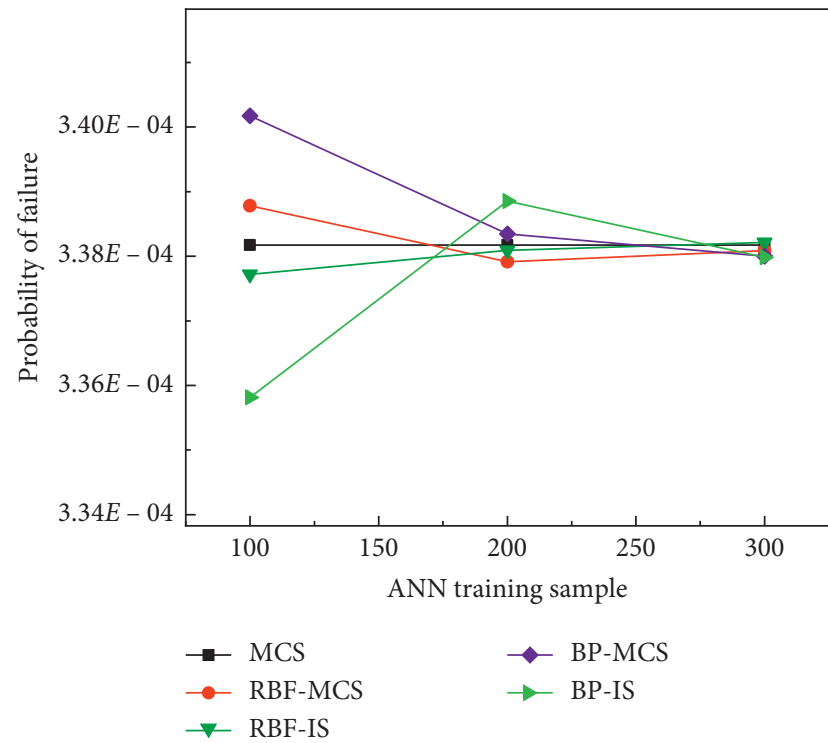

Figure 9: Comparison of the POF between ANN-IS (RBF-IS and BP-IS) and various other methods relative to ANN sample data size.

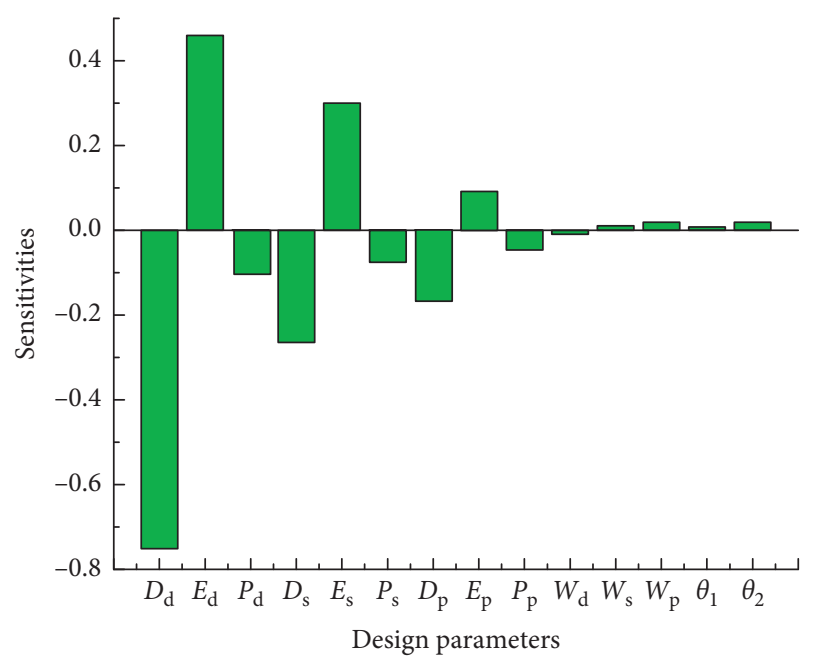

FIGURE 10: The result of sensitivity analysis.
TABle 8: The mean and standard deviation of the design parameters.

\begin{tabular}{lcccc}
\hline $\begin{array}{l}\text { Design } \\
\text { parameters }\end{array}$ & Mean & $\begin{array}{c}\text { Standard } \\
\text { deviation }\end{array}$ & Distribution & Units \\
\hline$x_{1}\left(D_{\mathrm{d}}\right)$ & 7330 & 366.5 & Normal & $\mathrm{kg} / \mathrm{m}^{3}$ \\
$x_{2}\left(E_{\mathrm{d}}\right)$ & $1.48 E+5$ & 7400 & Normal & $\mathrm{MPa}$ \\
$x_{3}\left(P_{\mathrm{d}}\right)$ & 0.31 & 0.0155 & Normal & - \\
$x_{4}\left(D_{\mathrm{s}}\right)$ & 2540 & 127 & Normal & $\mathrm{kg} / \mathrm{m}^{3}$ \\
$x_{5}\left(E_{\mathrm{s}}\right)$ & $1.0 E+5$ & 5000 & Normal & $\mathrm{MPa}$ \\
$x_{6}\left(P_{\mathrm{s}}\right)$ & 0.25 & 0.0125 & Normal & - \\
$x_{7}\left(D_{\mathrm{p}}\right)$ & 7100 & 355 & Normal & $\mathrm{kg} / \mathrm{m}^{3}$ \\
$x_{8}\left(E_{\mathrm{p}}\right)$ & $7.252 E+4$ & 3626 & Normal & $\mathrm{MPa}$ \\
$x_{9}\left(P_{\mathrm{p}}\right)$ & 0.32 & 0.016 & Normal & - \\
\hline
\end{tabular}

The POF of the RBF-IS method is $1.184 E-04$, $1.185 E-04$, and $1.184 E-04$ and that of the BP-IS method is $1.187 E-04,1.185 E-04$, and $1.184 E-04$, respectively. The results show that the $\mathrm{COV}$ (POF) values of different methods are less than $3 E-2$, and the calculated results from different reliability analysis methods are basically consistent. The POF of the RBF-IS method relative to change ANN sample data size is smaller than those of other methods. In addition, the ANN-IS method has better calculation efficiency than the ANN-based MCS method from number of samples. The number of samples of the ANN-based MCS method is $1 E+7$ and that of the ANN-IS method is $1 E+4$.

\section{Discussion and Conclusions}

This research evaluated the calculation accuracy and efficiency of the ANN-IS method on reliability of structures such as drum brakes. By analyzing the results of two numeric verification problems and the drum brake vibration reliability problem, we arrive at the following conclusions.

First, the result of the RBF-IS method is closer to that of the MCS method than is the BP-IS method result. After reviewing the results of two numeric verification problems, the results calculated from the MCS method are found to be consistent with reference MCS results. The relative errors of the RBF-IS method for the two numeric verification problems are $0.0058 \%$ and $0.0136 \%$, respectively, which are smaller than the results from other methods for the same ANN sample size. 


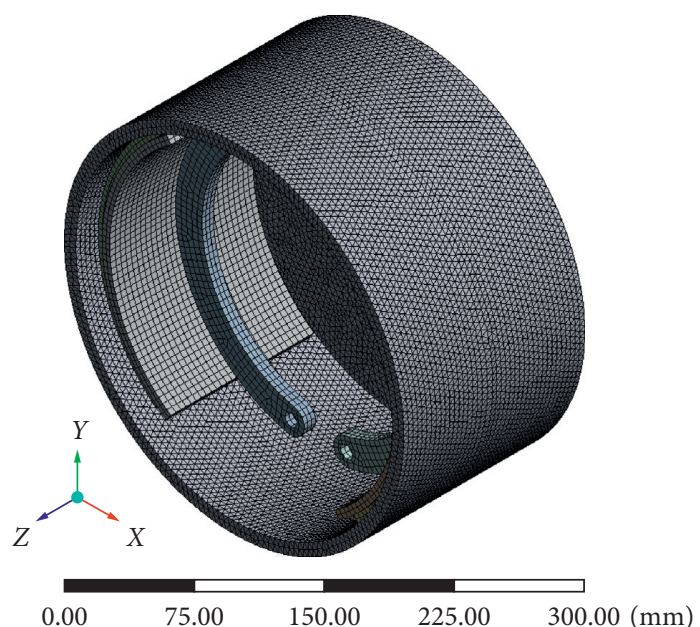

(a)

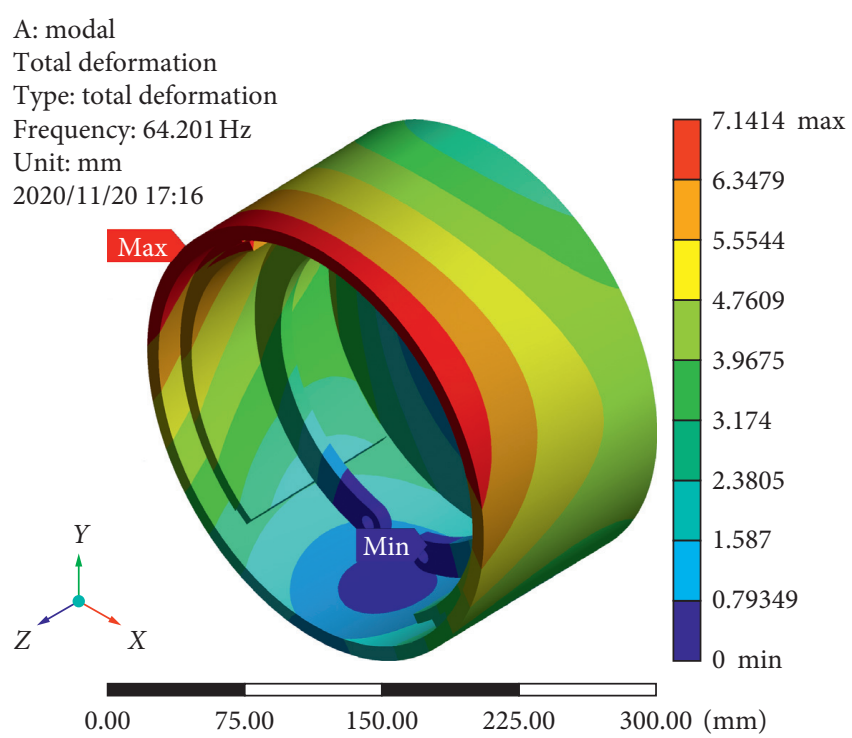

(b)

Figure 11: The FEA result of the drum brake model. (a) The mesh of the drum brake model. (b) The natural frequency of the drum brake.

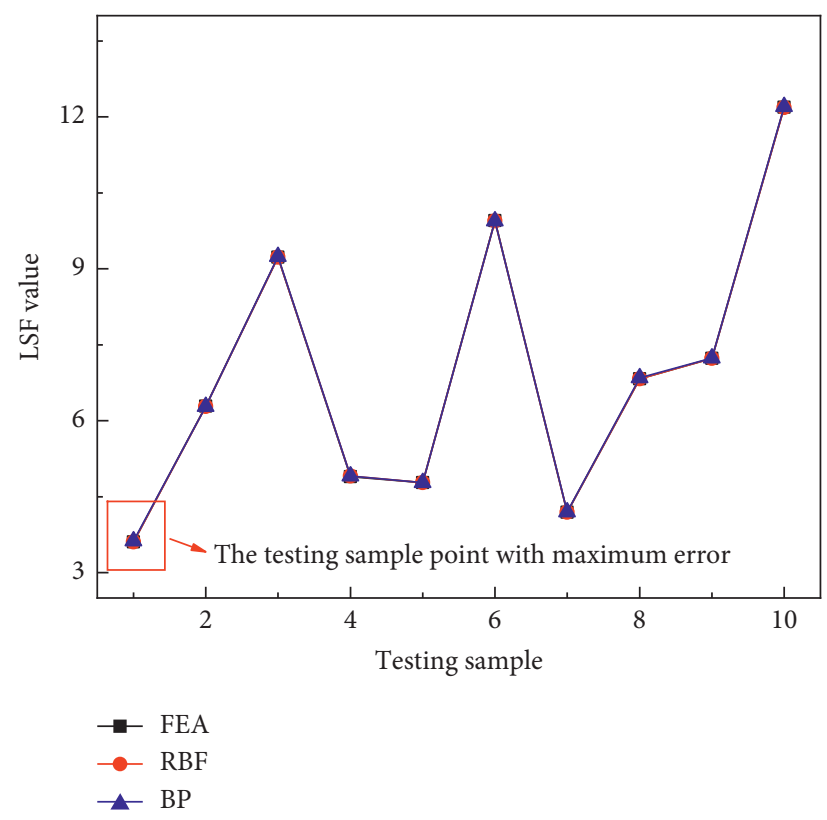

(a)

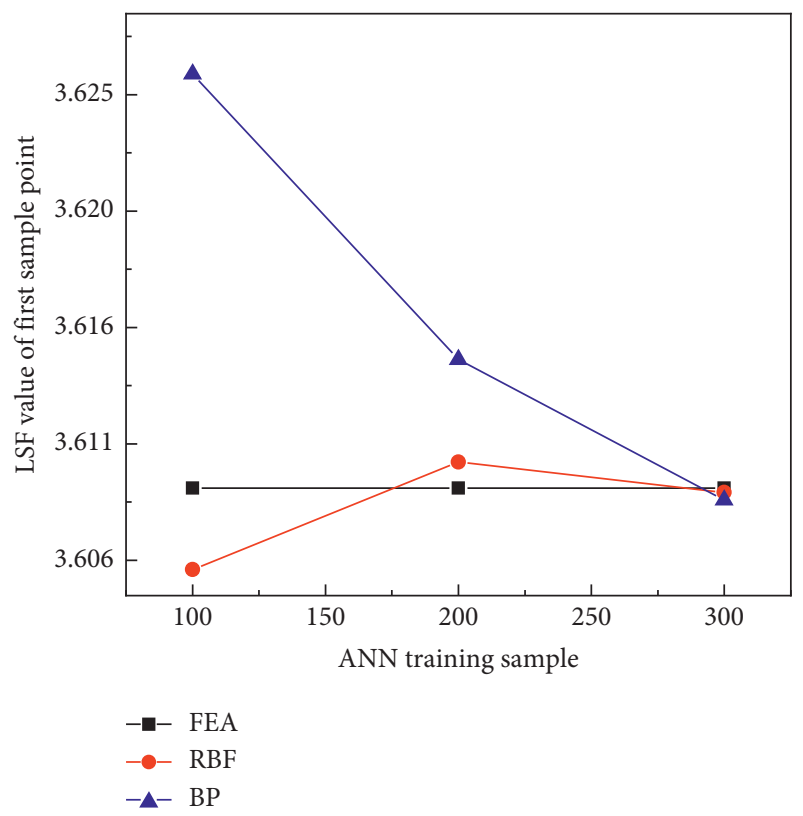

(b)

FIgUre 12: Comparison between the FEA values and the predicted LSF values by ANNs. (a) The LSF value at testing sample for an ANN sample size of 100. (b) The LSF value of the first testing sample point relative to change in the ANN sample data size.

Second, the RBF-IS method has a better calculation efficiency than the other methods used in this study. Through numeric analysis, find that use of the RBF-IS method results in smaller changes to the POF as the ANN sample size changes than when other methods are used. Additionally, the POF of the RBF-IS method at an ANN sample size of 200 is found to be closer to that of the MCS method than those of other methods (including BP-IS) at an ANN sample data size of 300 . For reliability of structures such as drum brake, the time required for the FEA increases with both the ANN sample size and the complexity of the structural model. Since the RBF-IS method can use a smaller ANN sample size than other methods while still main training good calculation accuracy, the required calculation time of the RBF-IS method is smaller than those of other methods (including BP-IS). Finally, the number of samples used by the RBF-IS method is $1 E+4$ and those used by the ANN-based MCS method is $1 E+7$. This indicates that the 


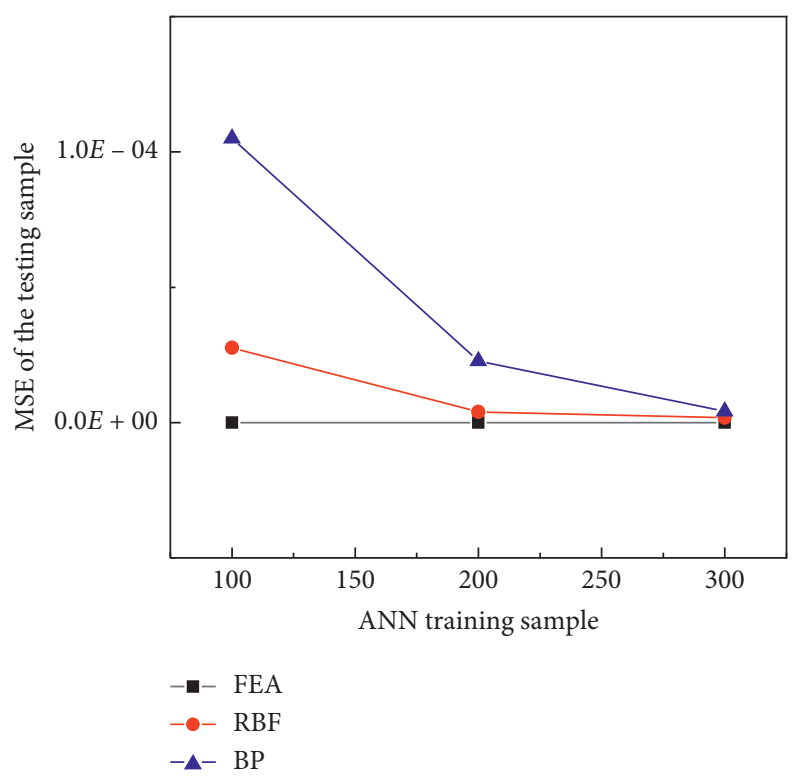

FIGURE 13: Comparison of the change of MSE between RBF and BP as the ANN sample data size change.

TABLE 9: Comparison of the reliability index and POF from different reliability analysis methods with an ANN sample size of 100.

\begin{tabular}{lcccr}
\hline Method & Reliability index & POF & Number of samples $\left(N_{1}\right)$ & COV $($ POF $)$ \\
\hline BP-MCS & 3.6768 & $1.181 E-04$ & $1 E+7$ & $2.910 E-02$ \\
BP-AFOSM & 3.7002 & $1.077 E-04$ & - & - \\
BP-IS & 3.6756 & $1.187 E-04$ & $1 E+4$ & $2.164 E-02$ \\
RBF-MCS & 3.6768 & $1.181 E-04$ & $1 E+7$ & $2.910 E-02$ \\
RBF-AFOSM & 3.6779 & $1.176 E-04$ & - & - \\
RBF-IS & 3.6762 & $1.184 E-04$ & $1 E+4$ & $2.176 E-02$ \\
\hline
\end{tabular}

TABle 10: Comparison of the reliability index and POF from different reliability analysis methods with an ANN sample size of 200.

\begin{tabular}{lcccr}
\hline Method & Reliability index & POF & Number of samples $\left(N_{1}\right)$ & COV $($ POF $)$ \\
\hline BP-MCS & 3.6766 & $1.182 E-04$ & $1 E+7$ & $2.908 E-02$ \\
BP-AFOSM & 3.7011 & $1.073 E-04$ & - & - \\
BP-IS & 3.6759 & $1.185 E-04$ & $1 E+4$ & $2.230 E-02$ \\
RBF-MCS & 3.6763 & $1.183 E-04$ & $1 E+7$ & $2.901 E-02$ \\
RBF-AFOSM & 3.6983 & $1.085 E-04$ & - & - \\
RBF-IS & 3.6760 & $1.185 E-04$ & $1 E+4$ & $2.194 E-02$ \\
\hline
\end{tabular}

TABLE 11: Comparison of the reliability index and POF from different reliability analysis methods with an ANN sample size of 300 .

\begin{tabular}{|c|c|c|c|c|}
\hline Method & Reliability index & POF & Number of samples $\left(N_{1}\right)$ & $\mathrm{COV}(\mathrm{POF})$ \\
\hline BP-MCS & 3.6761 & $1.184 E-04$ & $1 E+7$ & $2.906 E-02$ \\
\hline BP-AFOSM & 3.7020 & $1.069 E-04$ & - & - \\
\hline BP-IS & 3.6762 & $1.184 E-04$ & $1 E+4$ & $2.218 E-02$ \\
\hline RBF-MCS & 3.6761 & $1.184 E-04$ & $1 E+7$ & $2.906 E-02$ \\
\hline RBF-AFOSM & 3.7040 & $1.061 E-04$ & - & - \\
\hline RBF-IS & 3.6761 & $1.184 E-04$ & $1 E+4$ & $2.226 E-02$ \\
\hline
\end{tabular}




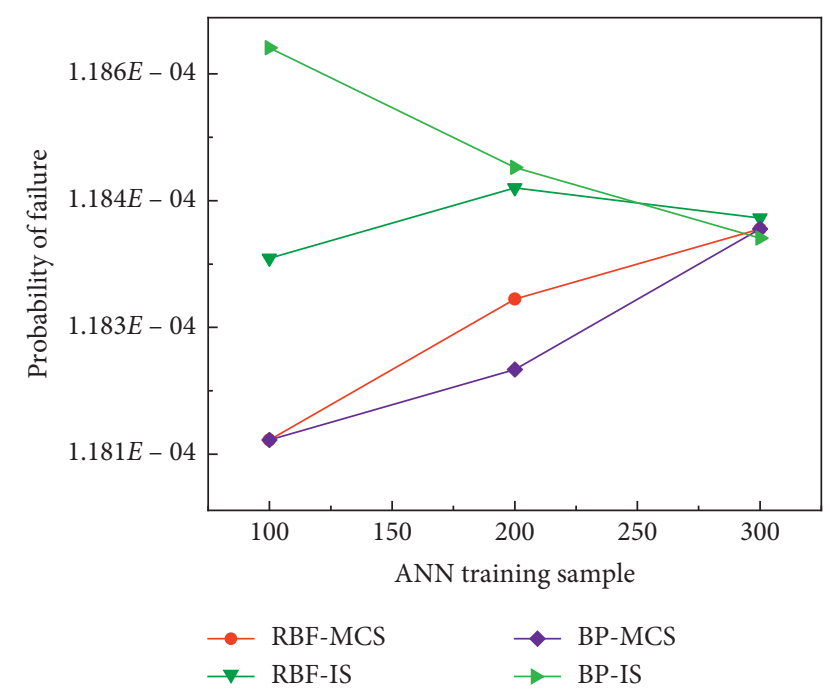

Figure 14: Comparison of the POF between ANN-IS (RBF-IS and BP-IS) and various other methods relative to ANN sample data size.

RBF-IS method offers a better calculation efficiency than the ANN-based MCS method.

The disadvantage of the RBF-IS method is that an optimization algorithm such as AFOSM is required to calculate the most probable point of the LSF, which requires the explicit form of the LSF to be known in advance.

Based on this research, find that the RBF-IS method is well suited to reliability problems such as the drum brake vibration reliability problem.

\section{Data Availability}

The datasets used to support the findings of this research are included within the Supplementary Materials.

\section{Additional Points}

In this study, according to the theory of the vibration reliability analysis, the ANN-IS method is used to conduct the vibration reliability analysis on the drum brake. In addition, the calculation results of two ANN-IS methods (RBF-IS and BP-IS) are compared for structure reliability problems. This research demonstrates that the RBF-IS method is well suited to structure reliability problems such as the drum brake vibration reliability problem.

\section{Conflicts of Interest}

The authors declare that there are no conflicts of interest.

\section{Authors' Contributions}

Z. Yang and U. S. Pak conceptualized the study; U. S. Pak and C. U. Kwon performed finite element analysis; U. S. Pak performed reliability analysis; Z. Yang, U. S. Pak, and C. U. Kwon reviewed and edited the article. All authors have read and agreed to the published version of the manuscript.

\section{Acknowledgments}

The authors are grateful for the support from Chinese National Natural Science Foundation (U1710119 and U1708254) and Fundamental Research Funds for the Central Universities (N2003022).

\section{Supplementary Materials}

Supplementary Data 1. Pseudocode of the proposed method. Supplementary Data 2. ANN sample data and testing data of the drum brake. Table 1. FEA results of drum brake when ANN sample data size is 100 . Table 2. FEA results of drum brake when ANN sample data size is 200. Table 3. FEA results of drum brake when ANN sample data size is 300 . Table 4. FEA results of ANN testing sample data. (Supplementary Materials)

\section{References}

[1] Y. Zhang, H. Zhang, and C. Lu, "Study on parameter optimization design of drum brake based on hybrid cellular multiobjective genetic algorithm," Mathematical Problems in Engineering, vol. 2012, Article ID 734193, 23 pages, 2012.

[2] M. Zhou, Y. Wang, and Q. Huang, "Study on the stability of drum brake non-linear low frequency vibration model," Archive of Applied Mechanics, vol. 77, no. 7, pp. 473-483, 2007.

[3] I. Ahmed, "Modeling of vehicle drum brake for contact analysis using ansys," SAE Technical Paper Series, vol. 7, 2012.

[4] A. Haldar and S. Mahadevan, "First-order and second-order reliability methods," Probabilistic Structural Mechanics Handbook, pp. 27-52, 1995.

[5] D. M. Pedroso, "Form reliability analysis using a parallel evolutionary algorithm," Structural Safety, vol. 65, pp. 84-99, 2017.

[6] F. Homaei and M. Najafzadeh, "A reliability-based probabilistic evaluation of the wave-induced scour depth around marine structure piles," Ocean Engineering, vol. 196, Article ID 106818, 2020.

[7] J. Zhang and X. Du, "A second-order reliability method with first-order efficiency," Journal of Mechanical Design, vol. 132, no. $10,2010$.

[8] X. Huang, Y. Li, and Y. Zhang, "A new direct second-order reliability analysis method," Applied Mathematical Modelling, vol. 55, pp. 68-80, 2018.

[9] A. Hosni Elhewy, E. Mesbahi, and Y. Pu, "Reliability analysis of structures using neural network method," Probabilistic Engineering Mechanics, vol. 21, no. 1, pp. 44-53, 2006.

[10] E. Gobet and P. Turkedjiev, "Adaptive importance sampling in least-squares Monte Carlo algorithms for backward stochastic differential equations," Stochastic Processes and their Applications, vol. 127, no. 4, pp. 1171-1203, 2017.

[11] I. Papaioannou, C. Papadimitriou, and D. Straub, "Sequential importance sampling for structural reliability analysis," Structural Safety, vol. 62, pp. 66-75, 2016.

[12] P. Bjerager, "Probability integration by directional simulation," Journal of Engineering Mechanics, vol. 114, pp. 12851302, 1988.

[13] D. A. Alvarez, F. Uribe, and J. E. Hurtado, "Estimation of the lower and upper bounds on the probability of failure using subset simulation and random set theory," Mechanical Systems and Signal Processing, vol. 100, pp. 782-801, 2018. 
[14] A. P. van den Eijnden and M. A. Hicks, "Efficient subset simulation for evaluating the modes of improbable slope failure," Computers and Geotechnics, vol. 88, pp. 267-280, 2017.

[15] M. Najafzadeh and F. Saberi-Movahed, "GMDH-GEP to predict free span expansion rates below pipelines under waves," Marine Georesources \& Geotechnology, vol. 37, no. 3, pp. 375-392, 2018.

[16] A. Hadidi, B. F. Azar, and A. Rafiee, "Efficient response surface method for high-dimensional structural reliability analysis," Structural Safety, vol. 68, pp. 15-27, 2017.

[17] X.-J. Meng, S.-K. Jing, L.-X. Zhang et al., "A new sampling approach for response surface method based reliability analysis and its application," Advances in Mechanical Engineering, vol. 7, no. 1, Article ID 305473, 2014.

[18] T. Zhang, X. P. Zhou, and X. F. Liu, "Reliability analysis of slopes using the improved stochastic response surface methods with multicollinearity," Engineering Geology, vol. 271, 2020.

[19] H. Zhao, Z. Ru, X. Chang et al., "Reliability analysis of tunnel using least square support vector machine," Tunnelling and Underground Space Technology, vol. 41, pp. 14-23, 2014.

[20] L.-F. You, J.-G. Zhang, S. Zhou et al., "A novel mixed uncertainty support vector machine method for structural reliability analysis," Acta Mechanica, vol. 232, no. 4, pp. 1497-1513, 2021.

[21] K. Cheng and Z. Lu, "Structural reliability analysis based on ensemble learning of surrogate models," Structural Safety, vol. 83, 2020.

[22] K. Yuan, N.-C. Xiao, Z. Wang et al., "System reliability analysis by combining structure function and active learning kriging model," Reliability Engineering \& System Safety, vol. 195, 2020.

[23] S. Xiao, S. Oladyshkin, and W. Nowak, "Reliability analysis with stratified importance sampling based on adaptive Kriging," Reliability Engineering \& System Safety, vol. 197, Article ID 106852, 2020.

[24] X. Zhang, L. Wang, and J. D. Sørensen, “AKOIS: an adaptive Kriging oriented importance sampling method for structural system reliability analysis," Structural Safety, vol. 82, 2020.

[25] H. M. Gomes and A. M. Awruch, "Comparison of response surface and neural network with other methods for structural reliability analysis," Structural Safety, vol. 26, no. 1, pp. 49-67, 2004.

[26] J. Deng, D. Gu, X. Li et al., "Structural reliability analysis for implicit performance functions using artificial neural network," Structural Safety, vol. 27, no. 1, pp. 25-48, 2005.

[27] Y. Hu, C.-d. Xiao, and Y.-y. Shi, "Reliability analysis for highly non-linear and complex model using ANN-MCM simulation," Journal of the Brazilian Society of Mechanical Sciences and Engineering, vol. 40, no. 5, 2018.

[28] Q. Wang and H. Fang, "Reliability analysis of tunnels using an adaptive RBF and a first-order reliability method," Computers and Geotechnics, vol. 98, pp. 144-152, 2018.

[29] J. Cheng, Q. S. Li, and R.-c. Xiao, "A new artificial neural network-based response surface method for structural reliability analysis," Probabilistic Engineering Mechanics, vol. 23, no. 1, pp. 51-63, 2008

[30] A. A. Chojaczyk, A. P. Teixeira, L. C. Neves et al., "Review and application of Artificial Neural Networks models in reliability analysis of steel structures," Structural Safety, vol. 52, pp. 78-89, 2015.

[31] P. A. M. Lopes, H. M. Gomes, and A. M. Awruch, "Reliability analysis of laminated composite structures using finite elements and neural networks," Composite Structures, vol. 92, no. 7, pp. 1603-1613, 2010.

[32] Y. Zhou, P. Unsong, and Z. Haoyuan, "Reliability sensitivity analysis of stochastic resonance failure of vehicle drum brake," in Proceedings of the 2020 5th International Conference on Electromechanical Control Technology and Transportation (ICECTT), Nanchang, China, 2020.

[33] C. Su, Y. Zhang, and Q. Zhao, "Vibration reliability sensitivity analysis of general system with correlation failure modes," Journal of Mechanical Science and Technology, vol. 25, no. 12, pp. 3123-3133, 2012.

[34] Y. Mo, S. Guo, and C. Tang, "A vibration reliability analysis method for the uncertain space beam structure," Shock and Vibration, vol. 2016, Article ID 9218590, 14 pages, 2016.

[35] Z. Yang, L. Panxue, W. Hao et al., "Frequency reliability-based sensitivity analysis of motorized spindle by BP neural networks," Journal of Harbin Institute of Technology, vol. 49, no. 1, pp. 30-36, 2017.

[36] C. Kwon, U. Song, U. Pak et al., "Effect of structural parameters on the flow field and power consumption of in-line high shear mixer," Journal of the Indian Chemical Society, vol. 98, no. 3, 2021.

[37] W. Li, F. Xia, S. Zhao et al., "Mixing performance of an inline high-shear mixer with a novel pore-array liquid distributor," Industrial \& Engineering Chemistry Research, vol. 58, no. 44, pp. 20213-20225, 2019. 